\title{
Averages of short exponential sums, II
}

by

Alexandru Zaharescu (Bucureşti and Urbana, IL)

1. Introduction. Exponential sums have proved to be very useful in many problems in number theory. The most powerful results obtained so far in this area are concerned with exponential sums in finite fields. There are many important results on this topic in the literature (see Weil [9], Bombieri [2], [3], Deligne [4], [5], Serre [8], Katz [6]). The theory works fine for complete exponential sums. By a standard procedure of expressing an incomplete exponential sum in terms of complete ones, it also works for incomplete sums. However, the quality of such results depends on the size of the range of summation of the given incomplete exponential sum, and it is getting worse when this size is small.

A technique devised to estimate certain averages of short exponential sums is presented in [10]. To be precise, let $\varepsilon>0$, let $N \leq M \leq P$ be positive integers and let $r(X)=f(X) / g(X)$ be a rational function which is not a polynomial, with integer coefficients bounded by $P^{K_{1}}$ and with $\operatorname{deg} f, \operatorname{deg} g<K_{2}$, where $K_{1}$ and $K_{2}$ are some positive constants. Then for almost all pairs $(p, m)$ with $p$ prime, $p \in[P, 2 P]$ and $m \in\{1, \ldots, M\}$ one has

$$
\left|\sum_{1 \leq n \leq N}^{*} e\left(\frac{m r(n)}{p}\right)\right| \ll_{\varepsilon, K_{1}, K_{2}} N^{1 / 2} P^{\varepsilon}
$$

Here and in what follows "almost all" means that the exceptional set has density $<P^{-\varepsilon}, r(n)$ is computed modulo $p$ and $\sum^{*}$ denotes a sum over values of $n$ for which $g(n)$ is nonzero modulo $p$. The above result was obtained via the following second moment estimate. Under the same assumptions one has

$$
\sum_{P \leq p \leq 2 P} \sum_{1 \leq m \leq M}\left|\sum_{1 \leq n \leq N}^{*} e\left(\frac{m r(n)}{p}\right)\right|^{2} \ll_{\varepsilon, K_{1}, K_{2}} N M P^{1+\varepsilon}
$$

2000 Mathematics Subject Classification: 11L07, 11L05, 11T23. 
In order to prove (1.2) one brings into play the pair correlation of the sets

$$
\mathcal{N}_{p}=\left\{\frac{r(n)(\bmod p)}{p}: 1 \leq n \leq N\right\}
$$

and use them to provide upper bounds for the short moments:

$$
M_{2}(N, M, r, p)=\sum_{1 \leq m \leq M}\left|\sum_{1 \leq n \leq N}^{*} e\left(\frac{m r(n)}{p}\right)\right|^{2}
$$

for each $p$ individually. Next, an alternative way of estimating the pair correlations is introduced, which produces the required amount of cancellation in these pair correlations when $p$ varies in the interval $[P, 2 P]$. Thus the point was to average the correlations rather than the exponential sums.

In this paper we present a generalization of the above results to rational functions of several variables $r\left(X_{1}, \ldots, X_{k}\right)$. Here the new feature is the appearance of an obstruction caused by a certain Diophantine equation, the size of the set of solutions of which controls the size of our exponential sums. Quite interestingly, this is a Diophantine equation over $\mathbb{Z}$ as is the case with our original rational function $r\left(X_{1}, \ldots, X_{k}\right)$, although the exponential sums under consideration are defined modulo $p$, with $p$ varying in an interval $[P, 2 P]$. More precisely, given a rational function $r\left(X_{1}, \ldots, X_{k}\right) \in \mathbb{Q}\left(X_{1}, \ldots, X_{k}\right)$ we consider, in the affine space $\mathbb{A}^{2 k+1}$, the hypersurface $H_{r}$ which consists of points $\left(x_{1}, \ldots, x_{k}, y_{1}, \ldots, y_{k}, z\right)$ satisfying the equation

$$
z=r\left(x_{1}, \ldots, x_{k}\right)-r\left(y_{1}, \ldots, y_{k}\right) .
$$

We call $H_{r}$ the pair correlation hypersurface associated to $r$. For any $k$-tuple $\left(N_{1}, \ldots, N_{k}\right)$ of positive integers define

$$
\begin{aligned}
\mathcal{A}\left(r, N_{1}, \ldots, N_{k}\right)=\left\{\left(x_{1}, \ldots, x_{k}, y_{1}, \ldots, y_{k}, z\right)\right. & \in \mathbb{Z}^{2 k+1} \cap H_{r}: \\
1 & \left.\leq x_{j}, y_{j} \leq N_{j}, 1 \leq j \leq k\right\} .
\end{aligned}
$$

Note that (1.3) has "diagonal solutions" $z=0, x_{1}=y_{1}, \ldots, x_{k}=y_{k}$, therefore $\mathcal{A}\left(r, N_{1}, \ldots, N_{k}\right)$ has at least $N_{1} \ldots N_{k}$ elements. Under the assumption that the number of integer solutions to (1.3) is not much larger than the number of diagonal solutions we obtain a generalization of (1.2) which again produces square root cancellation on average in the corresponding exponential sums.

THEOREM 1. Let $\varepsilon>0$, let $N_{1}, \ldots, N_{k} \leq P$ and $M$ be positive integers and let $r(X)=f\left(X_{1}, \ldots, X_{k}\right) / g\left(X_{1}, \ldots, X_{k}\right)$ be a rational function with integer coefficients bounded by $P^{K_{1}}$ and with $\operatorname{deg} f \operatorname{deg} g<K_{2}$, where $K_{1}$ and $K_{2}$ are some positive constants. Assume that

$$
\# \mathcal{A}\left(r, N_{1}, \ldots, N_{k}\right) \ll_{\varepsilon, k, K_{1}, K_{2}} N_{1} \ldots N_{k} P^{\varepsilon} .
$$


Then

$$
\begin{aligned}
\sum_{P \leq p \leq 2 P} \sum_{1 \leq m \leq M}\left|\sum_{1 \leq n_{1} \leq N_{1}} \ldots \sum_{1 \leq n_{k} \leq N_{k}}^{*} e\left(\frac{m r\left(n_{1}, \ldots, n_{k}\right)}{p}\right)\right|^{2} \\
\ll_{\varepsilon, k, K_{1}, K_{2}} N_{1} \ldots N_{k}\left(M P+P N_{1} \ldots N_{k}+N_{1} \ldots N_{k} M\right) P^{\varepsilon} .
\end{aligned}
$$

As a consequence, if $N_{1} \ldots N_{k} \leq \min \{P, M\}$ then we get square root cancellation on average in the above exponential sums:

Corollary 1. Under the hypotheses of Theorem 1, if $N_{1} \ldots N_{k} \leq$ $\min \{P, M\}$ then for almost all pairs $(p, m)$ with $p$ prime, $p \in[P, 2 P]$ and $m \in\{1, \ldots, M\}$ one has

$$
\begin{aligned}
&\left|\sum_{1 \leq n_{1} \leq N_{1}} \ldots \sum_{1 \leq n_{k} \leq N_{k}}^{*} e\left(\frac{m r\left(n_{1}, \ldots, n_{k}\right)}{p}\right)\right| \\
& \ll_{\varepsilon, k, K_{1}, K_{2}} N_{1}^{1 / 2} \ldots N_{k}^{1 / 2} P^{\varepsilon} .
\end{aligned}
$$

In practice, in order to apply the above results one first needs to check whether the given rational function $r\left(X_{1}, \ldots, X_{k}\right)$ satisfies the above condition (1.4). Let us remark that if two rational functions $r_{1}\left(X_{1}, \ldots, X_{k}\right)$ and $r_{2}\left(X_{1}, \ldots, X_{k}\right)$ differ by a polynomial, that is,

$$
r_{1}\left(X_{1}, \ldots, X_{k}\right)-r_{2}\left(X_{1}, \ldots, X_{k}\right) \in \mathbb{Z}\left[X_{1}, \ldots, X_{k}\right],
$$

then for any $k$-tuple $\left(N_{1}, \ldots, N_{k}\right)$ one has

$$
\# \mathcal{A}\left(r_{1}, N_{1}, \ldots, N_{k}\right)=\# \mathcal{A}\left(r_{2}, N_{1}, \ldots, N_{k}\right) .
$$

Therefore (1.4) holds for $r_{1}\left(X_{1}, \ldots, X_{k}\right)$ if and only if it holds for $r_{2}\left(X_{1}, \ldots\right.$ $\left.\ldots, X_{k}\right)$. In case $k=1$ the relation (1.4) holds for any $r(X)$ which is not a polynomial (see [10], Section 3). For functions of several variables this is no longer the case. For example, if $k \geq 2$ and $b$ is a nonzero integer then (1.4) fails for the rational function

$$
r\left(X_{1}, \ldots, X_{k}\right)=\frac{b}{X_{1}+\ldots+X_{k}} .
$$

Indeed, in this case any $(2 k+1)$-tuple $\left(x_{1}, \ldots, x_{k}, y_{1}, \ldots, y_{k}, 0\right)$ with $x_{1}, \ldots$ $\ldots, x_{k}, y_{1}, \ldots, y_{k} \in \mathbb{Z}, 1 \leq x_{j}, y_{j} \leq N_{j}$ for $1 \leq j \leq k$ and $x_{1}+\ldots+x_{k}=$ $y_{1}+\ldots+y_{k}$ will be an element of $\mathcal{A}\left(r, N_{1}, \ldots, N_{k}\right)$. If we replace the sum from the denominator of the right hand side of (1.7) by the product $X_{1} \ldots X_{k}$ then the condition (1.4) will hold true. By the above remark this condition will continue to hold if a polynomial is added to our rational function. In particular if we take

$$
r\left(X_{1}, \ldots, X_{k}\right)=a_{1} X_{1}+\ldots+a_{k} X_{k}+\frac{b}{X_{1} \ldots X_{k}}
$$


then (1.4) holds and we obtain square root cancellation in certain averages of short hyper-Kloosterman sums:

Corollary 2. Let $K, \varepsilon>0$, let $P, M, N_{1}, \ldots, N_{k}$ be positive integers such that $N_{1} \ldots N_{k}<\min \{P, M\}$ and let $b \neq 0$ and $a_{1}, \ldots, a_{k}$ be integers bounded by $P^{K}$. Then for almost all pairs $(p, m)$ with $p$ prime, $p \in[P, 2 P]$ and $1 \leq m \leq M$ one has

$$
\begin{array}{r}
\left|\sum_{1 \leq n_{1} \leq N_{1}} \ldots \sum_{1 \leq n_{k} \leq N_{k}} e\left(\frac{m\left(a_{1} n_{1}+\ldots+a_{k} n_{k}+b \overline{n_{1} \ldots n_{k}}\right)}{p}\right)\right| \\
\ll_{\varepsilon, k, K} N_{1}^{1 / 2} \ldots N_{k}^{1 / 2} P^{\varepsilon},
\end{array}
$$

where $\overline{n_{1} \ldots n_{k}}$ denotes the inverse of $n_{1} \ldots n_{k}$ modulo $p$.

As was pointed out by the referee, our results have a similarity to the Large Sieve Inequality. In fact, in case $r(n)=n$ (which is not covered by our results) the left hand side of (1.2) can be written in the form $\sum_{j=1}^{R}\left|S\left(\alpha_{j}\right)\right|^{2}$, where $S(\alpha)=\sum_{n=1}^{N} e(n \alpha)$ and $\left\{\alpha_{1}, \ldots, \alpha_{R}\right\}$ is the set of fractions $m / p$ with $1 \leq m \leq M$ and $P \leq p \leq 2 P, p$ prime. The Large Sieve Inequality (see Montgomery [7]) gives an upper bound of the form

$$
\sum_{j=1}^{R}\left|S\left(\alpha_{j}\right)\right|^{2} \leq \Delta(N, \delta) \sum_{n=M+1}^{M+N}\left|a_{n}\right|^{2}
$$

for any trigonometric polynomial with complex coefficients

$$
S(\alpha)=\sum_{n=M+1}^{M+N} a_{n} e(n \alpha)
$$

and any real numbers $\alpha_{1}, \ldots, \alpha_{R}$ which are well spaced $(\bmod 1)$ in the sense that $\left\|\alpha_{j}-\alpha_{s}\right\| \geq \delta$ for $j \neq s$, where $\|\cdot\|$ denotes the distance to the nearest integer. Here one can take $\Delta(N, \delta)=N-1+\delta^{-1}$. Our method also works with weights $a_{n}$ or, in general, $a\left(n_{1}, \ldots, n_{k}\right)$ attached to the sums. The results are presented in Section 4.4 below.

Acknowledgements. The author is grateful to the referee whose comments led to the results presented in Section 4.4.

2. Exponential sums and pair correlations. Let $\mathcal{N}=\left\{x_{n}: 1 \leq\right.$ $n \leq N\}$ be a finite sequence of points in the interval $[0,1]$ and let $M$ be a positive integer. An upper bound for the second moment

$$
M_{2}(\mathcal{N}, M):=\sum_{1 \leq m \leq M}\left|\sum_{1 \leq n \leq N} e\left(m x_{n}\right)\right|^{2}
$$


is provided in [10], Section 2, in terms of the pair correlation of the set $\mathcal{N}$. The result is

$$
M_{2}(\mathcal{N}, M) \ll M E(\mathcal{N}, M)
$$

where

$$
E(\mathcal{N}, M)=\#\left\{1 \leq n, n^{\prime} \leq N:\left\|x_{n}-x_{n^{\prime}}\right\| \leq 1 / M\right\} .
$$

Now let $r\left(X_{1}, \ldots, X_{k}\right)$ be a rational function as in the statement of Theorem 1 and let $p$ be a prime number in the interval $[P, 2 P]$. Choose positive integers $N_{1}, \ldots, N_{k}$ less than $p$ and let $B\left(r, p, N_{1}, \ldots, N_{k}\right)$ be the set of $k$-tuples $\left(n_{1}, \ldots, n_{k}\right)$ of positive integers with $n_{j} \leq N_{j}, 1 \leq j \leq k$, for which $g\left(n_{1}, \ldots, n_{k}\right)$ is nonzero modulo $p$. Denote by $\mathcal{N}\left(r, p, N_{1}, \ldots, N_{k}\right)$ the set of values of $r\left(n_{1}, \ldots, n_{k}\right)(\bmod p) / p$, counted with multiplicities, as $\left(n_{1}, \ldots, n_{k}\right)$ varies in $B\left(r, p, N_{1}, \ldots, N_{k}\right)$. From (2.1) applied to $\mathcal{N}=$ $\mathcal{N}\left(r, p, N_{1}, \ldots, N_{k}\right)$ we get

$$
\begin{aligned}
& \sum_{1 \leq m \leq M}\left|\sum_{1 \leq n_{1} \leq N_{1}} \ldots \sum_{1 \leq n_{k} \leq N_{k}}^{*} e\left(\frac{m r\left(n_{1}, \ldots, n_{k}\right)}{p}\right)\right|^{2} \\
& \ll M E\left(\mathcal{N}\left(r, p, N_{1}, \ldots, N_{k}\right), M\right)
\end{aligned}
$$

where

$$
\begin{aligned}
& E\left(\mathcal{N}\left(r, p, N_{1}, \ldots, N_{k}\right), M\right) \\
& =\#\left\{\left(n_{1}, \ldots, n_{k}\right),\left(n_{1}^{\prime}, \ldots, n_{k}^{\prime}\right) \in B\left(r, p, N_{1}, \ldots, N_{k}\right):\right. \\
& \left.\quad r\left(n_{1}, \ldots, n_{k}\right)-r\left(n_{1}^{\prime}, \ldots, n_{k}^{\prime}\right) \equiv h(\bmod p),|h| \leq p / M\right\} .
\end{aligned}
$$

3. Averaging over $p$. We add the inequalities (2.2) for all primes $p \in$ $[P, 2 P]$ to obtain

$$
\begin{aligned}
& \sum_{P \leq p \leq 2 P} \sum_{1 \leq m \leq M} \mid \sum_{1 \leq n_{1} \leq N_{1}}\left.\ldots \sum_{1 \leq n_{k} \leq N_{k}}^{*} e\left(\frac{m r\left(n_{1}, \ldots, n_{k}\right)}{p}\right)\right|^{2} \\
& \ll M \sum_{P \leq p \leq 2 P} E\left(\mathcal{N}\left(r, p, N_{1}, \ldots, N_{k}\right), M\right) .
\end{aligned}
$$

Note that the sum on the right hand side equals the number of $(2 k+2)$ tuples $\left(n_{1}, \ldots, n_{k}, n_{1}^{\prime}, \ldots, n_{k}^{\prime}, h, p\right)$ satisfying the following conditions:

$p$ prime, $\quad P \leq p \leq 2 P$,

$h \in \mathbb{Z}, \quad|h| \leq p / M$,

$$
\begin{aligned}
& n_{1}, \ldots, n_{k}, n_{1}^{\prime}, \ldots, n_{k}^{\prime} \in \mathbb{Z}, \\
& 1 \leq n_{j}, n_{j}^{\prime} \leq N_{j}, \quad 1 \leq j \leq k, \\
& g\left(n_{1}, \ldots, n_{k}\right), g\left(n_{1}^{\prime}, \ldots, n_{k}^{\prime}\right) \text { nonzero }(\bmod p), \\
& r\left(n_{1}, \ldots, n_{k}\right)-r\left(n_{1}^{\prime}, \ldots, n_{k}^{\prime}\right) \equiv h(\bmod p) .
\end{aligned}
$$


The last condition in (3.2) is equivalent to

$$
\begin{aligned}
g\left(n_{1}^{\prime}, \ldots, n_{k}^{\prime}\right) f\left(n_{1}, \ldots, n_{k}\right) & -f\left(n_{1}^{\prime}, \ldots, n_{k}^{\prime}\right) g\left(n_{1}, \ldots, n_{k}\right) \\
& \equiv h g\left(n_{1}^{\prime}, \ldots, n_{k}^{\prime}\right) g\left(n_{1}, \ldots, n_{k}\right)(\bmod p) .
\end{aligned}
$$

For any solution $\left(n_{1}, \ldots, n_{k}, n_{1}^{\prime}, \ldots, n_{k}^{\prime}, h, p\right)$ to $(3.2)$ consider the integer

$$
\begin{aligned}
A= & g\left(n_{1}^{\prime}, \ldots, n_{k}^{\prime}\right) f\left(n_{1}, \ldots, n_{k}\right)-f\left(n_{1}^{\prime}, \ldots, n_{k}^{\prime}\right) g\left(n_{1}, \ldots, n_{k}\right) \\
& -h g\left(n_{1}^{\prime}, \ldots, n_{k}^{\prime}\right) g\left(n_{1}, \ldots, n_{k}\right) .
\end{aligned}
$$

We count separately the solutions with $A=0$ and those with $A \neq 0$. Let $\left(n_{1}, \ldots, n_{k}, n_{1}^{\prime}, \ldots, n_{k}^{\prime}, h, p\right)$ be a solution with $A=0$. Then

$$
r\left(n_{1}, \ldots, n_{k}\right)-r\left(n_{1}^{\prime}, \ldots, n_{k}^{\prime}\right)=h
$$

in $\mathbb{Q}$, hence $\left(n_{1}, \ldots, n_{k}, n_{1}^{\prime}, \ldots, n_{k}^{\prime}, h\right)$ is an integer point on the hypersurface $H_{r}$. Moreover, from (3.2) we see that this point belongs to $\mathcal{A}\left(r, N_{1}, \ldots, N_{k}\right)$. By our assumption (1.4), the number of elements of $\mathcal{A}\left(r, N_{1}, \ldots, N_{k}\right)$ is $\ll_{\varepsilon, k, K_{1}, K_{2}} N_{1} \ldots N_{k} P^{\varepsilon}$. Any such $(2 k+1)$-tuple $\left(n_{1}, \ldots, n_{k}, n_{1}^{\prime}, \ldots, n_{k}^{\prime}, h\right)$ appears in less than $P$ solutions $\left(n_{1}, \ldots, n_{k}, n_{1}^{\prime}, \ldots, n_{k}^{\prime}, h, p\right)$ to $(3.2)$, therefore the number of solutions with $A=0$ is

$$
\ll_{\varepsilon, k, K_{1}, K_{2}} N_{1} \ldots N_{k} P^{1+\varepsilon} .
$$

We now count the solutions to $(3.2)$ for which $A \neq 0$. Choose a $(2 k+1)$ tuple $\left(n_{1}, \ldots, n_{k}, n_{1}^{\prime}, \ldots, n_{k}^{\prime}, h\right)$ which appears in such a solution. The point here is that $A$ cannot have too many prime divisors. More precisely, from the hypotheses of Theorem 1 we see that

$$
A \ll_{k, K_{2}} P^{2 K_{1}}\left(\max \left\{N_{1}, \ldots, N_{k}\right\}\right)^{2 K_{2}} \max \{P / M, 1\} \ll P^{2 K_{1}+2 K_{2}+1} .
$$

It follows that the number of primes $p \in[P, 2 P]$ which divide $A$ is bounded by a constant which depends on $k, K_{1}$ and $K_{2}$ only.

Taking into account that by $(3.2)$ the $(2 k+1)$-tuple $\left(n_{1}, \ldots, n_{k}, n_{1}^{\prime}, \ldots\right.$ $\left.\ldots, n_{k}^{\prime}, h\right)$ takes at most $N_{1}^{2} \ldots N_{k}^{2} \max \{2 P / M, 1\}$ values, we find that the number of solutions to $(3.2)$ with $A \neq 0$ is

$$
\ll_{\varepsilon, k, K_{1}, K_{2}} \frac{N_{1}^{2} \ldots N_{k}^{2}(P+M)}{M} .
$$

Therefore the total number of solutions to (3.2) is

$$
\ll_{\varepsilon, k, K_{1}, K_{2}} N_{1} \ldots N_{k} P^{1+\varepsilon}+\frac{N_{1}^{2} \ldots N_{k}^{2}(P+M)}{M} .
$$

Using this bound for the sum on the right hand side of (3.1) we obtain (1.5), which completes the proof of Theorem 1.

\section{Further remarks, applications and generalizations}

4.1. In order to prove Corollary 2 we need to check that $r\left(X_{1}, \ldots, X_{k}\right)$ $=b /\left(X_{1} \ldots X_{k}\right)$ satisfies $(1.4)$. Let us count the number of $(2 k+1)$-tuples 
$\left(x_{1}, \ldots, x_{k}, y_{1}, \ldots, y_{k}, z\right)$ in $\mathcal{A}\left(r, N_{1}, \ldots, N_{k}\right)$ with $x_{1}, \ldots, x_{k}$ fixed. Multiplying (1.3) by $x_{1} \ldots x_{k}$ we find that $b x_{1} \ldots x_{k} /\left(y_{1} \ldots y_{k}\right)$ is an integer. This almost fixes $y_{1}, \ldots, y_{k}$ since the number of divisors of the nonzero integer $b x_{1} \ldots x_{k}$ is $O_{\varepsilon, k, K_{1}}\left(P^{\varepsilon}\right)$. Next, with $\left(x_{1}, \ldots, x_{k}, y_{1}, \ldots, y_{k}\right)$ fixed, $z$ is uniquely determined by (1.3). Thus $r\left(X_{1}, \ldots, X_{k}\right)$ satisfies (1.4).

4.2. Under the hypotheses of Corollary 1 , assume also that all the elements $\left(x_{1}, \ldots, x_{k}, y_{1}, \ldots, y_{k}, z\right)$ of $\mathcal{A}\left(r, N_{1}, \ldots, N_{k}\right)$ satisfy

$$
|z|<P / M \text {. }
$$

Note that for any $\left(x_{1}, \ldots, x_{k}, y_{1}, \ldots, y_{k}, z\right)$ in $\mathcal{A}\left(r, N_{1}, \ldots, N_{k}\right)$ one has

$$
\begin{aligned}
|z| & \leq\left|f\left(x_{1}, \ldots, x_{k}\right)\right|+\left|f\left(y_{1}, \ldots, y_{k}\right)\right| \\
& \leq 2 P^{K_{1}}\left(K_{2}+1\right)^{k}\left(\max \left\{N_{1}, \ldots, N_{k}\right\}\right)^{K_{2}},
\end{aligned}
$$

therefore (4.1) will hold if we assume that $K_{1}<1$ and

$$
P^{1-K_{1}}>2 M\left(\max \left\{N_{1}, \ldots, N_{k}\right\}\right)^{K_{2}} .
$$

Under the above assumptions the condition (1.4) besides being sufficient is also necessary in order to have square root cancellation on average in the exponential sums under consideration. Indeed, on the one hand by an appropriate modification of the argument which gives (2.1) one also obtains an upper bound for $M E(\mathcal{N}, M)$ in terms of exponential sums:

$$
M E(\mathcal{N}, M) \ll \sum_{|m| \leq M}\left|\sum_{1 \leq n \leq N} e\left(m x_{n}\right)\right|^{2}
$$

(see for example Chapter 2 of Baker [1]). This provides an upper bound for the sum on the right hand side of (3.1), and hence also for the number, call it $l$, of solutions of the system (3.2), in terms of exponential sums. On the other hand, following the proof of Theorem 1 and using also (4.1) we see that each element of $\mathcal{A}\left(r, N_{1}, \ldots, N_{k}\right)$ in combination with each admissible prime $p$ produce a solution to (3.2), and this gives a lower bound for $l$. Now if $r\left(X_{1}, \ldots, X_{k}\right)$ does not satisfy (1.4), this lower bound will be too large to still have square root cancellation on average in the exponential sums appearing in the above upper bound for $l$.

4.3. As in Theorem 3 of [10], we may combine Theorem 1 above with the Erdős-Turán inequality to obtain a square root saving on average in the discrepancy of the sets

$$
\begin{aligned}
\mathcal{N}\left(r, p, m, N_{1}, \ldots, N_{k}\right):=\left\{m r\left(n_{1}, \ldots, n_{k}\right) / p: 1 \leq n_{j} \leq N_{j}, 1 \leq j \leq k,\right. \\
\left.g\left(n_{1}, \ldots, n_{k}\right) \neq 0(\bmod p)\right\} .
\end{aligned}
$$

The result is 
THEOREM 2. Under the hypotheses of Theorem 1, assume also that $N_{1} \ldots$ $\ldots N_{k} \leq \min \{P, M\}$ and that (1.4) holds with $r$ replaced by $m r$ for any $1 \leq$ $m \leq N_{1} \ldots N_{k}$. Then for almost all pairs $(p, m)$ with $p$ prime, $p \in[P, 2 P]$ and $1 \leq m \leq M$ one has

$$
D\left(\mathcal{N}\left(r, p, m, N_{1}, \ldots, N_{k}\right)\right) \ll_{\varepsilon, k, K_{1}, K_{2}} N_{1}^{1 / 2} \ldots N_{k}^{1 / 2} P^{\varepsilon} .
$$

Here the discrepancy $D(\mathcal{N})$ of a finite sequence $\mathcal{N}=\left\{x_{n}: 1 \leq n \leq N\right\}$ of points in $[0,1]$ is defined by

$$
D(\mathcal{N})=\sup _{0 \leq \alpha<\beta \leq 1}|\#(\mathcal{N} \cap[\alpha, \beta])-N(\beta-\alpha)|
$$

The proof of the above theorem goes along the same lines as that of Theorem 3 of [10]. As a consequence of Theorem 2 we have the following

COROLlary 3. Under the hypotheses of Theorem 2, for almost all pairs $(p, m)$ with $p$ prime, $p \in[P, 2 P]$ and $1 \leq m \leq M$ and for any $\beta \in[0,1]$ there exist $1 \leq n_{1} \leq N_{1}, \ldots, 1 \leq n_{k} \leq N_{k}$ such that

$$
\left|\left\{\frac{m r\left(n_{1}, \ldots, n_{k}\right)(\bmod p)}{p}\right\}-\beta\right| \ll_{\varepsilon, k, K_{1}, K_{2}} N_{1}^{-1 / 2} \ldots N_{k}^{-1 / 2} P^{\varepsilon} .
$$

4.4. One can generalize the inequalities (1.2) and (1.5) by attaching weights $a_{n}$, respectively $a\left(n_{1}, \ldots, n_{k}\right)$ to the corresponding exponential sums. We are looking for an upper bound for the sum

$$
S:=\sum_{P \leq p \leq 2 P} \sum_{|m| \leq M}\left|\sum_{1 \leq n_{j} \leq N_{j}, 1 \leq j \leq k}^{*} a\left(n_{1}, \ldots, n_{k}\right) e\left(\frac{m r\left(n_{1}, \ldots, n_{k}\right)}{p}\right)\right|^{2}
$$

where the weights $a\left(n_{1}, \ldots, n_{k}\right)$ are arbitrary complex numbers. In this case instead of the number of elements in the set $\mathcal{A}\left(r, N_{1}, \ldots, N_{k}\right)$ we consider the weighted sum

$$
\sigma=\sum_{(\mathbf{x}, \mathbf{y}, z) \in \mathcal{A}\left(r, N_{1}, \ldots, N_{k}\right)}\left|a\left(x_{1}, \ldots, x_{k}\right) a\left(y_{1}, \ldots, y_{k}\right)\right|
$$

where we have set $\mathbf{x}=\left(x_{1}, \ldots, x_{k}\right), \mathbf{y}=\left(y_{1}, \ldots, y_{k}\right)$. One has the following generalization of Theorem 1 .

Theorem 3. Let $K_{1}, K_{2}, \varepsilon>0$, let $N_{1}, \ldots, N_{k} \leq P$ and $M$ be positive integers, let $a\left(n_{1}, \ldots, n_{k}\right)$ with $1 \leq n_{j} \leq N_{j}, 1 \leq j \leq k$, be complex numbers and let $r(X)=f\left(X_{1}, \ldots, X_{k}\right) / g\left(X_{1}, \ldots, X_{k}\right)$ be a rational function with integer coefficients bounded by $P^{K_{1}}$ and with $\operatorname{deg} f, \operatorname{deg} g \leq K_{2}$. Then

$$
|S| \ll_{\varepsilon, k, K_{1}, K_{2}} P M \sigma+P^{\varepsilon}(P+M)\left(\sum_{1 \leq n_{j} \leq N_{j}, 1 \leq j \leq k}\left|a\left(n_{1}, \ldots, n_{k}\right)\right|\right)^{2} .
$$


Taking into account the contribution of the diagonal terms $\mathbf{x}=\mathbf{y}$ in (4.2) we see that one always has

$$
\sigma \geq \sum_{1 \leq n_{j} \leq N_{j}, 1 \leq j \leq k}\left|a\left(n_{1}, \ldots, n_{k}\right)\right|^{2} .
$$

In case $k=1$ and $r(X)=f(X) / g(X)$ is not a polynomial, reasoning in terms of the resultant $R(f, g)$ as in Section 3 of [10] one finds that $\sigma$ is not much larger than the right hand side of (4.4); more precisely,

$$
\sigma \ll_{\varepsilon, K_{1}, K_{2}} P^{\varepsilon} \sum_{1 \leq n \leq N}\left|a_{n}\right|^{2} .
$$

Hence we obtain the following result.

Theorem 4. Let $K_{1}, K_{2}, \varepsilon>0$, let $N \leq P$ and $M$ be positive integers, let $a_{n}$ with $1 \leq n \leq N$ be complex numbers and let $r(X)=f(X) / g(X)$ be a rational function with integer coefficients bounded by $P^{K_{1}}$ and with $\operatorname{deg} f, \operatorname{deg} g \leq K_{2}, r(X)$ not a polynomial. Then

$$
\begin{aligned}
\sum_{P \leq p \leq 2 P} \sum_{|m| \leq M} \mid & \left.\sum_{1 \leq n \leq N}^{*} a_{n} e\left(\frac{m r(n)}{p}\right)\right|^{2} \\
& \ll_{\varepsilon, K_{1}, K_{2}} P^{1+\varepsilon}\left[M \sum_{1 \leq n \leq N}\left|a_{n}\right|^{2}+\left(\sum_{1 \leq n \leq N}\left|a_{n}\right|\right)^{2}\right] .
\end{aligned}
$$

Here we applied Cauchy's inequality in order to get rid of the term $P^{\varepsilon} M\left(\sum_{1 \leq n \leq N}\left|a_{n}\right|\right)^{2}$. Applying it one more time we obtain the following generalization of (1.2).

COROLlaRY 4. Under the hypotheses of Theorem 4, assume also that $N \leq M$. Then

$$
\sum_{P \leq p \leq 2 P} \sum_{|m| \leq M}\left|\sum_{1 \leq n \leq N}^{*} a_{n} e\left(\frac{m r(n)}{p}\right)\right|^{2} \ll_{\varepsilon, K_{1}, K_{2}} P^{1+\varepsilon} M \sum_{1 \leq n \leq N}\left|a_{n}\right|^{2} .
$$

Returning to Theorem 3, its proof uses the following lemma.

Lemma 1. For any real numbers $x_{1}, \ldots, x_{N}$, any complex numbers $a_{1}, \ldots$ $\ldots, a_{N}$ and any positive integer $M$ one has

$$
\sum_{|m| \leq M}\left|\sum_{1 \leq n \leq N} a_{n} e\left(m x_{n}\right)\right|^{2} \ll M \sum_{\substack{1 \leq n, n^{\prime} \leq N \\\left\|x_{n}-x_{n^{\prime}}\right\| \leq 1 / M}}\left|a_{n} a_{n^{\prime}}\right| .
$$

The proof of Lemma 1 goes along the same lines as the proof of (2.1) given in [10]: reasoning in terms of the real and imaginary part of $a_{n}$ we may assume that the $a_{n}$ 's are real; next, by putting together the positive, respectively negative weights one further reduces the problem to the case 
when the $a_{n}$ 's are positive numbers. Then the positivity argument from Section 2 of [10] works and the lemma is proved.

If we use Lemma 1 instead of (2.1) in the above relations (2.2) and (3.1) the result is that in $(3.2)$ we have to count each solution $\left(n_{1}, \ldots, n_{k}, n_{1}^{\prime}, \ldots\right.$ $\left.\ldots n_{k}^{\prime}, h, p\right)$ with a weight given by $\left|a\left(n_{1}, \ldots, n_{k}\right) a\left(n_{1}^{\prime}, \ldots, n_{k}^{\prime}\right)\right|$. Note that the congruence (3.3) does not depend on the weights, so we have the same two classes of solutions to (3.2) as before, according as $A=0$ or $A \neq 0$. The contribution of solutions with $A=0$ to the right hand side of (4.3) is captured in the term $P M \sigma$, while the contribution of solutions with $A \neq 0$ is bounded by the other term, and this proves Theorem 3 .

\section{References}

[1] R. C. Baker, Diophantine Inequalities, Oxford Univ. Press, New York, 1986.

[2] E. Bombieri, On exponential sums in finite fields, Amer. J. Math. 88 (1966), 71-105.

[3] -, On exponential sums in finite fields. II, Invent. Math. 47 (1978), 29-39.

[4] P. Deligne, La conjecture de Weil. I, Inst. Hautes Études Sci. Publ. Math. 43 (1974), 273-307.

[5] —, La conjecture de Weil. II, ibid. 52 (1980), 137-252.

[6] N. M. Katz, Sommes exponentielles, Astérisque 79 (1980).

[7] H. L. Montgomery, The analytic principle of the large sieve, Bull. Amer. Math. Soc. 84 (1978), 547-567.

[8] J. P. Serre, Majoration de sommes exponentielles, Astérisque 41-42 (1977), 111-126.

[9] A. Weil, On some exponential sums, Proc. Nat. Acad. Sci. U.S.A. 34 (1948), 204207.

[10] A. Zaharescu, Averages of short exponential sums, Acta Arith. 88 (1999), 223-231.

Institute of Mathematics of the Romanian Academy

P.O. Box 1-764

RO-70700 Bucureşti, Romania
Department of Mathematics University of Illinois at Urbana-Champaign Altgeld Hall 1409 W. Green Street Urbana, IL 61801, U.S.A. E-mail: zaharesc@math.uiuc.edu 